\title{
X-ray phase-contrast tomography of breast tissue specimen with a multi-aperture analyser synchrotron set-up.
}

\author{
M. Endrizzi, ${ }^{a, 1}$ F.A. Vittoria ${ }^{a}$ L. Brombal ${ }^{b, c}$ R. Longo ${ }^{b, c}$ F. Zanconati ${ }^{d}$ A. Olivo ${ }^{a}$ \\ ${ }^{a}$ Department of Medical Physics and Biomedical Engineering, University College London, Gower Street, \\ London WC1E 6BT, United Kingdom \\ ${ }^{b}$ Dipartimento di Fisica, Università degli Studi di Trieste, Via A. Valerio 2, 34127 Trieste, Italy \\ ${ }^{c}$ Istituto Nazionale di Fisica Nucleare (INFN), Sezione di Trieste, Via A. Valerio, 2, 34127 Trieste, Italy \\ ${ }^{d}$ Pathology and Cytology Unit, University Hospital of Trieste, Strada di Fiume 447, 34149 Trieste, Italy \\ E-mail: m.endrizzi@ucl.ac.uk
}

ABSTRACT: We report on the application of the multi-aperture analyser X-ray Phase-Contrast imaging (XPCI) technique to the three-dimensional imaging of breast tissue samples. The experiment was conducted at the SYRMEP beamline (Elettra synchrotron, Italy) with a monochromatic X-ray beam. Along with the presentation of the methodology and resulting images, the potential extension of this approach to enable in-vivo applications at acceptable doses is discussed.

KeYwords: Inspection with x-rays, X-ray mammography, X-ray radiography and digital radiography (DR)

\footnotetext{
${ }^{1}$ Corresponding author.
} 


\section{Contents}

1 Introduction $\quad 1$

2 Methods $r$

3 Results and Discussion $\quad 3$

4 Conclusion 4

\section{Introduction}

$\mathrm{X}$-ray imaging is a widely used method for the non-destructive inspection of the internal structure of bulk samples. Its applications are numerous, encompassing security screening, material science, biology and medicine. In conventional, absorption-based radiography, image contrast is generated by the differences in the absorption that the X-ray beam experiences while travelling across the sample. When the differences in absorption become small, X-ray phase-contrast imaging XPCI [1] techniques can be applied to enhance image quality.

Several approaches for XPCI, involving both synchrotron facilities and laboratory sources, have been proposed and investigated [2-17]. Here we focus on a multi-aperture analyser set-up [18] implemented with monochromatic synchrotron radiation. It can be viewed as a development of edge illumination (EI) [19], where the sensing edge is replaced by a set of apertures to simultaneously attain high sensitivity and wide dynamic range. The EI method was shown to provide quantitative phase and dark-field images [20,21], by means of synchrotron radiation, microfocus tubes and conventional rotating anode sources with extended focal spots [22,23]. It also proved to be robust against very relaxed coherence conditions and against thermal and mechanical instabilities [24-27] and enabled low-dose implementations of XPCI in planar and three-dimensional imaging [28-30].

\section{Methods}

The experimental set-up is depicted in Figure 1: a $20 \mathrm{keV}$ monochromatic X-ray beam is amplitudemodulated by a narrow slit in the vertical ( $y$-axis) direction. After passing through the sample, the shaped beam is analysed by a set of apertures that are placed just in front of the detector (Photonic Science Ltd. CCD featuring $12.5 \mu \mathrm{m}$ pixel size). In order to reconstruct three-dimensional volumes, 600 views of the sample are collected by rotating it around the $y$-axis, in angular steps of 0.3 degrees. The illumination function (IF), i.e. the function that describes how the detected intensity changes as a function of the vertical misalignment $\bar{y}$ between the pre-sample aperture and the detector apertures, is sampled at 5 different positions. The modifications imparted by the sample to the illumination function are used to retrieve the sample absorption and refraction. 


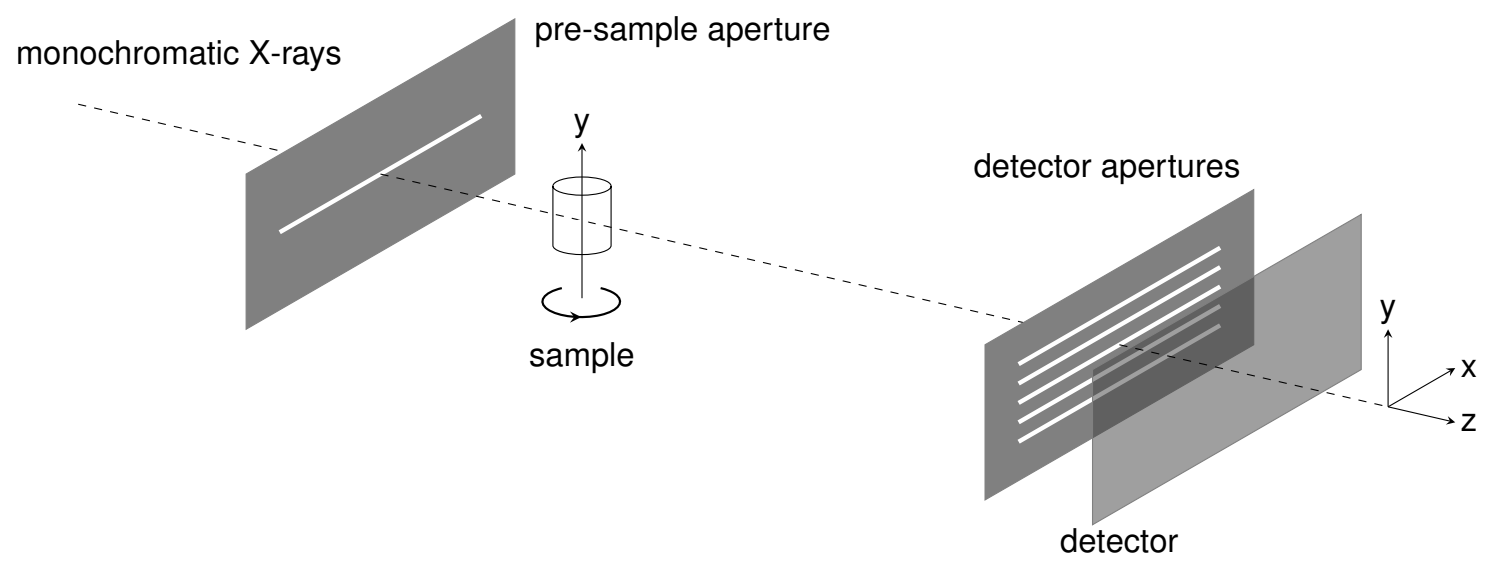

Figure 1: Schematic of the experimental set-up. A monochromatic X-ray beam is shaped by an absorbing aperture in the vertical (y) direction, it is passed through a sample that can translate along and rotate around the $y$-axis, and it is analysed by a set of apertures before detection.

This is achieved by expressing the measured intensity as a convolution between the IF $L(\bar{y})$ and the sample function $O(x, y)[18]$ :

$$
I(\bar{y})=\int L(\bar{y}-y) O(y) d y .
$$

which can be further expanded by using a multi-Gaussian model [21]:

$$
I(\bar{y})=\sum_{m} \sum_{n} A_{m n} \exp \left[-\frac{\left(\bar{y}-\mu_{m n}\right)^{2}}{2 \sigma_{m n}^{2}}\right]
$$

where $L(\bar{y})=\sum_{n}\left(A_{n} / \sqrt{2 \pi \sigma_{n}^{2}}\right) \exp \left[-\left(\bar{y}-\mu_{n}\right)^{2} / 2 \sigma_{n}^{2}\right],(n=1 \ldots N), O(\bar{y})=\sum_{m}\left(A_{m} / \sqrt{2 \pi \sigma_{m}^{2}}\right)$ $\exp \left[-\left(\bar{y}-\mu_{m}\right)^{2} / 2 \sigma_{m}^{2}\right],(m=1 \ldots M)$, and the parameters are defined in the following way: $\mu_{m n}=\mu_{m}+\mu_{n}, \sigma_{m n}^{2}=\sigma_{m}^{2}+\sigma_{n}^{2}$ and $A_{m n}=A_{m} A_{n}\left(1 / \sqrt{2 \pi \sigma_{m n}^{2}}\right)$. In this case $N=5$ terms were used for describing the IF.

The representations of the sample in the $(x, z)$ plane are obtained reconstructing the following two quantities by means of the Filtered Back Projection algorithm

$$
\begin{aligned}
-\ln (t) & =(4 \pi / \lambda) \int \beta(z) d z \\
\Delta y_{R} & =-z_{o d} \partial_{y} \int \delta(z) d z
\end{aligned}
$$

where $\delta$ indicates the decrement from unity of the refractive index, and $\beta$ its imaginary part.

In this proof-of-principle arrangement, featuring only one pre-sample aperture, only one slice at a time can be collected, then the sample has to be scanned along the $y$ direction and the acquisition procedure repeated.

The examined samples were derived from surgical material sent to the Pathology Unit according to local guidelines for histological examination of the Pathology Unit of the Academic Hospital 
of Cattinara, Trieste University, accredited by JCI (Joint Commission International). The images presented in this study were acquired from specimens of breast lumpectomy obtained out of overleft material to be wasted. The samples contain ductal carcinoma, glandular and adipose tissues. The samples were fixed in $4 \%$ neutral buffered formalin and sealed in cylindrical polyethylene containers ( 2.5 and $3 \mathrm{~cm}$ in diameter).

The work reported in this paper was carried out following the Directive 2004/23/EC of the European Parliament and of the Council of 31 March 2004 on setting standards of quality and safety for the donation, procurement, testing, processing, preservation, storage and distribution of human tissues.

Prior to the tomographic reconstruction, the absorption sinograms were filtered to mitigate ring artefacts due to gain inhomogeneities of the CCD. The applied ring-removal procedure is based on a combined wavelet-Fourier de-striping filtering, first proposed by Munch et al. [31], with a decomposition level of 5 and a width of the Gaussian bandpass function of 2 .

\section{Results and Discussion}

In Figure 2 slices from two samples are shown, with the conventional, absorption-based tomograms in the left-hand side column, and the refraction tomograms on the right-hand side.

In the absorption images, glandular and cancer regions components are hyperintense compared to the adipose component and the subtle glandular structures in adipose are perfectly visible. The refraction images offer a high-contrast visualization of the interface between different types of tissue. Moreover, in the refraction images the signal texture is different in glandular and in adipose regions, further studies are necessary, comparing tomographic images and histology in order to understand the origin of this texture differences and to evaluate their possible diagnostic significance in terms of presence of microvasculature or specific disorder in the cancer cell organization. Several artefacts are visible in those images, they can be mainly attributed to the presence of strong-contrast edges due to air bubbles within the samples.

The dose delivered to the samples was evaluated only afterwards, and was estimaed in the order of $17 \mathrm{~Gy}$. This value is clearly too high for clinical implementations of the technique, however several hardware and data acquisition strategy modifications can be considered, that would signifincalty reduce it. By keeping the same statistics at the detector, a factor of 20 can be gained by using a direct conversion device with approximately $100 \mu$ m pixels and detection efficiency approaching $90 \%$, like that used in the SYRMEP breast CT development $[32,33]$ (which doubles the detection efficiency of the scintillator used in this experiment). A further reduction by a factor of 5 can be achieved by using smaller apertures in the masks [28]. Moreover, instead of sampling the illumination function in 5 points, single-shot retrieval algorithms can be used [34,35]. These modifications alone would already reduce the dose to a few tens of mGy, at which point further optimization of the energy, exposure time per projection and possibly of the reconstruction algorithms would enable meeting the low-dose requirements of clinical implementation [36-39]. 


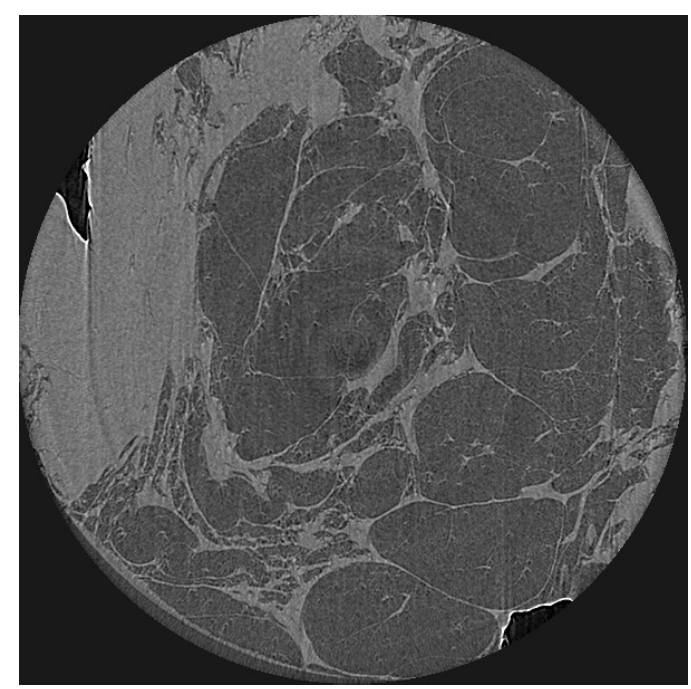

(a)

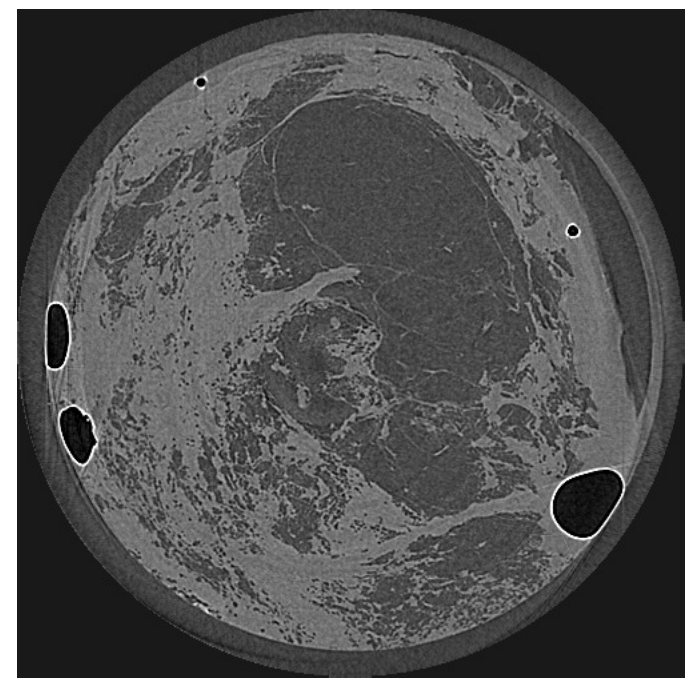

(c)

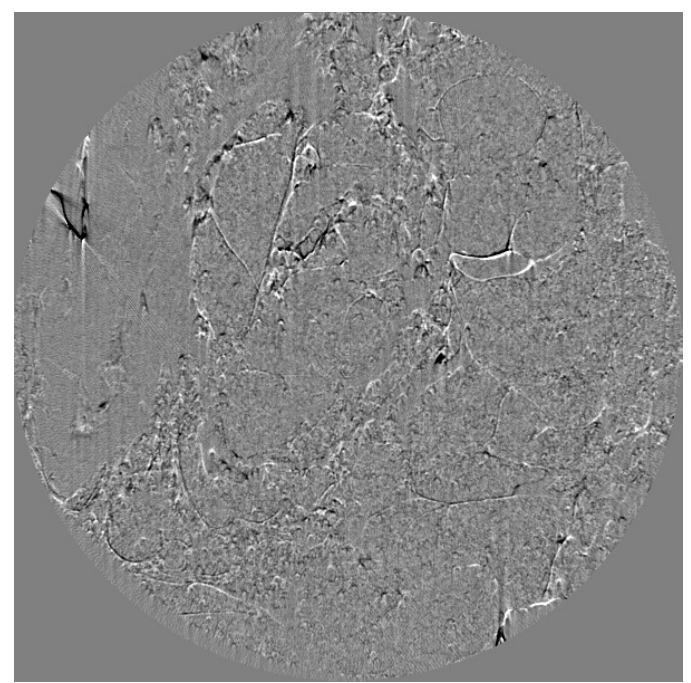

(b)

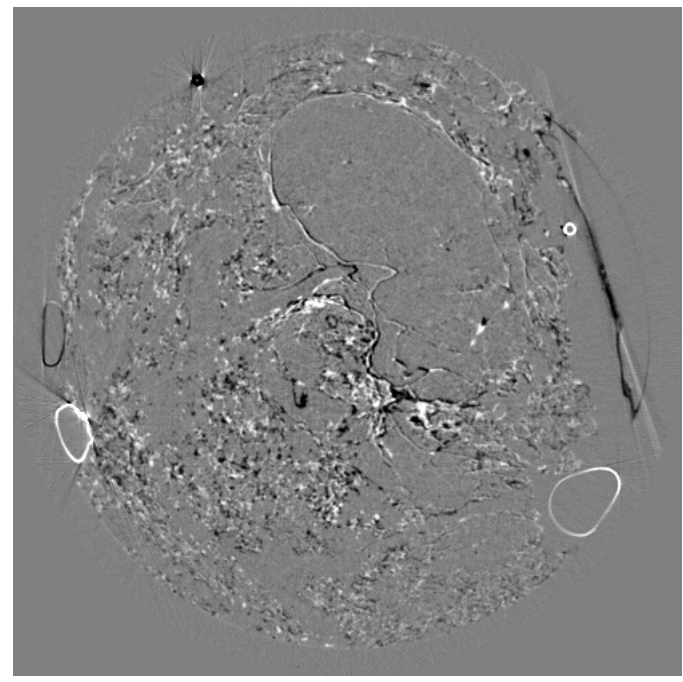

(d)

Figure 2: Two example slices: (a) and (c) are the absorption tomograms, while (b) and (d) are the refraction tomograms. Images (a) and (b) have $3 \mathrm{~cm}$ diameter, while (c) and (d) have $2.5 \mathrm{~cm}$ diameter.

\section{Conclusion}

Breast tissue samples where imaged through a multi-aperture analyser set-up using monochromatic synchrotron radiation. Absorption and refraction tomograms were successfully reconstructed for samples of approximately 2.5 and $3 \mathrm{~cm}$ diameters. A ring-removal procedure was used to attenuate ring-shaped artefacts in the absorption slices. Streak-type artefacts were present in the refraction images and are mainly attributed to the presence of air bubbles within the sample. Finally, strategies to reduce the dose delivered to the sample were proposed, which could enable moving the technique towards clinical applications in the future. 


\section{Acknowledgments}

ME was supported by the Royal Academy of Engineering under the RAEng Research Fellowships scheme. FAV is supported by the EPSRC (Grant EP/M507970/1). We thank Elettra Sincrotrone Trieste for access to SYRMEP beamline (proposal 20140147) that contributed to the results presented here.

\section{References}

[1] M. Endrizzi, X-ray phase-contrast imaging, Nuclear Instruments and Methods in Physics Research Section A: Accelerators, Spectrometers, Detectors and Associated Equipment 878 (2018) 88 - 98.

[2] U. Bonse and M. Hart, An x-ray interferometer, Applied Physics Letters 6 (1965) 155 -156.

[3] K. Goetz, E. Foerster, P. Zaumseil, M. P. Kalashnikov, I. A. Mikhailov, G. V. Sklizkov et al., Measurements of the parameters of shell targets for laser thermonuclear fusion using an X-ray schlieren method, Kvantovaia Elektronika Moscow 6 (1979) 1037-1042.

[4] T. J. Davis, D. Gao, T. E. Gureyev, A. W. Stevenson and S. W. Wilkins, Phase-contrast imaging of weakly absorbing materials using hard X-rays, Nature 373 (1995) 595-598.

[5] V. N. Ingal and E. A. Beliaevskaya, X-ray plane-wave topography observation of the phase contrast from a non-crystalline object, Journal of Physics D Applied Physics 28 (Nov., 1995) 2314-2317.

[6] S. W. Wilkins, T. E. Gureyev, D. Gao, A. Pogany and A. W. Stevenson, Phase-contrast imaging using polychromatic hard x-rays, Nature 384 (1996) 335-338.

[7] D. Chapman, W. Thomlinson, R. E. Johnston, D. Washburn, E. Pisano, N. Gmür et al., Diffraction enhanced x-ray imaging, Physics in Medicine and Biology 42 (1997) 2015-2025.

[8] J. F. Clauser, Us patent 5,812,629, 1998.

[9] C. David, B. Nohammer, H. H. Solak and E. Ziegler, Differential x-ray phase contrast imaging using a shearing interferometer, Applied Physics Letters 81 (2002) 3287-3289.

[10] A. Momose, S. Kawamoto, I. Koyama, Y. Hamaishi, K. Takai and Y. Suzuki, Demonstration of x-ray talbot interferometry, Japanese Journal of Applied Physics 42 (2003) L866.

[11] S. C. Mayo and B. Sexton, Refractive microlens array for wave-front analysis in the medium to hard $x$-ray range, Optics Letters 29 (2004) 866-868.

[12] F. Pfeiffer, T. Weitkamp, O. Bunk and C. David, Phase retrieval and differential phase-contrast imaging with low-brilliance X-ray sources, Nature Physics 2 (2006) 258-261.

[13] M. D. de Jonge, B. Hornberger, C. Holzner, D. Legnini, D. Paterson, I. McNulty et al., Quantitative phase imaging with a scanning transmission x-ray microscope, Physical Review Letters 100 (Apr, 2008) 163902.

[14] H. Wen, E. E. Bennett, M. M. Hegedus and S. Rapacchi, Fourier x-ray scattering radiography yields bone structural information1, Radiology 251 (June 2009) 910-918.

[15] K. S. Morgan, D. M. Paganin and K. K. W. Siu, X-ray phase imaging with a paper analyzer, Applied Physics Letters 100 (2012) 124102.

[16] H. Wang, Y. Kashyap and K. Sawhney, Hard-x-ray directional dark-field imaging using the speckle scanning technique, Physical Review Letters 114 (2015) 103901. 
[17] H. Miao, A. Panna, A. A. Gomella, E. E. Bennett, S. Znati, L. Chen et al., A universal moiré effect and application in $x$-ray phase-contrast imaging, Nature physics 12 (2016) 830-834.

[18] M. Endrizzi, F. A. Vittoria, L. Rigon, D. Dreossi, F. Iacoviello, P. R. Shearing et al., X-ray phase-contrast radiography and tomography with a multiaperture analyzer, Phys. Rev. Lett. 118 (Jun, 2017) 243902.

[19] A. Olivo, F. Arfelli, G. Cantatore, R. Longo, R. H. Menk, S. Pani et al., An innovative digital imaging set-up allowing a low-dose approach to phase contrast applications in the medical field, Medical Physics 28 (2001) 1610-1619.

[20] P. R. Munro, K. Ignatyev, R. D. Speller and A. Olivo, Phase and absorption retrieval using incoherent X-ray sources, Proc. Natl. Acad. Sci. USA 109 (2012) 13922-13927.

[21] M. Endrizzi, P. C. Diemoz, T. P. Millard, J. L. Jones, R. D. Speller, I. K. Robinson et al., Hard x-ray dark-field imaging with incoherent sample illumination, Applied Physics Letters 104 (2014) 024106.

[22] A. Olivo and R. Speller, A coded-aperture technique allowing $x$-ray phase contrast imaging with conventional sources, Applied Physics Letters 91 (2007) 074106.

[23] M. Endrizzi, F. A. Vittoria, P. C. Diemoz, R. Lorenzo, R. D. Speller, U. H. Wagner et al., Phase-contrast microscopy at high $x$-ray energy with a laboratory setup, Optics Letters 39 (2014) 3332-3335.

[24] P. R. T. Munro, K. Ignatyev, R. D. Speller and A. Olivo, Source size and temporal coherence requirements of coded aperture type $x$-ray phase contrast imaging systems, Optics Express 18 (2010) 19681.

[25] M. Endrizzi, F. A. Vittoria, G. Kallon, D. Basta, P. C. Diemoz, A. Vincenzi et al., Achromatic approach to phase-based multi-modal imaging with conventional $x$-ray sources, Optics Express 23 (2015) 16473-16480.

[26] T. P. Millard, M. Endrizzi, K. Ignatyev, C. K. Hagen, P. R. T. Munro, R. D. Speller et al., Method for automatization of the alignment of a laboratory based $x$-ray phase contrast edge illumination system, Review of Scientific Instruments 84 (2013) 083702.

[27] M. Endrizzi, D. Basta and A. Olivo, Laboratory-based x-ray phase-contrast imaging with misaligned optical elements, Applied Physics Letters 107 (2015) 124103.

[28] A. Olivo, S. Gkoumas, M. Endrizzi, C. K. Hagen, M. B. Szafraniec, P. C. Diemoz et al., Low-dose phase contrast mammography with conventional x-ray sources, Medical Physics 40 (2013) 090701.

[29] C. Hagen, P. Munro, M. Endrizzi, P. Diemoz and A. Olivo, Low-dose phase contrast tomography with conventional x-ray sources, Medical Physics 41 (2014) 070701.

[30] M. Longo, L. Rigon, F. C. M. Lopez, R. Chen, D. Dreossi, F. Zanconati et al., A simplified edge illumination set-up for quantitative phase contrast mammography with synchrotron radiation at clinical doses, Physics in Medicine and Biology 60 (2015) N21.

[31] B. Münch, P. Trtik, F. Marone and M. Stampanoni, Stripe and ring artifact removal with combined waveletâĂ Ťfourier filtering, Optics express 17 (2009) 8567-8591.

[32] R. Longo, F. Arfelli, R. Bellazzini, U. Bottigli, A. Brez, F. Brun et al., Towards breast tomography with synchrotron radiation at elettra: first images, Physics in medicine and biology 61 (2016) 1634.

[33] P. Delogu, B. Golosio, C. Fedon, F. Arfelli, R. Bellazzini, A. Brez et al., Imaging study of a phase-sensitive breast-ct system in continuous acquisition mode, Journal of Instrumentation 12 (2017) C01016. 
[34] P. C. Diemoz, F. A. Vittoria, C. K. Hagen, M. Endrizzi, P. Coan, E. Brun et al., Single-image phase retrieval using an edge illumination $x$-ray phase-contrast imaging setup, Journal of synchrotron radiation 22 (2015) 1072-1077.

[35] P. C. Diemoz, C. K. Hagen, M. Endrizzi, M. Minuti, R. Bellazzini, L. Urbani et al., Single-shot x-ray phase-contrast computed tomography with nonmicrofocal laboratory sources, Phys. Rev. Applied 7 (Apr, 2017) 044029.

[36] S. Vedantham, L. Shi, A. Karellas, A. M. O'Connell and D. L. Conover, Personalized estimates of radiation dose from dedicated breast ct in a diagnostic population and comparison with diagnostic mammography, Physics in medicine and biology 58 (2013) 7921.

[37] A. Sarno, G. Mettivier and P. Russo, Dedicated breast computed tomography: basic aspects, Medical physics 42 (2015) 2786-2804.

[38] B. Zhao, X. Zhang, W. Cai, D. Conover and R. Ning, Cone beam breast ct with multiplanar and three dimensional visualization in differentiating breast masses compared with mammography, European journal of radiology 84 (2015) 48-53.

[39] W. A. Kalender, D. Kolditz, C. Steiding, V. Ruth, F. Lück, A.-C. Rößler et al., Technical feasibility proof for high-resolution low-dose photon-counting ct of the breast, European radiology 27 (2017) 1081-1086. 